\section{ORIGINAL RESEARCH}

N.Z. Hobbs

J. Barnes

C. Frost

S.M.D. Henley

E.J. Wild

K. Macdonald
R.A. Barker
R.I. Scahill
N.C. Fox

S.J. Tabrizi

\title{
Onset and Progression of Pathologic Atrophy in Huntington Disease: A Longitudinal MR Imaging Study
}

BACKGROUND AND PURPOSE: Longitudinal MR imaging measures provide an opportunity to track progression in HD before the emergence of clinical symptoms. This may prove useful in assessing disease-modifying treatments. We investigated how caudate and global volumes change as HD progresses from premanifest to early disease.

MATERIALS AND METHODS: Forty HD gene-positive individuals and 19 controls underwent serial volumetric MR imaging (baseline, 12 and 27 months; 2 or 3 scans per person). At baseline, 3 patients with HD were premanifest but developed overt motor features during the study, and 37 had early HD. All had dates of motor onset recorded. Caudates, lateral ventricles, and TIVs were measured using semiautomated procedures. Linear mixed models were used to investigate differences between HD and controls in relation to motor onset, controlling for TIV, sex, and age.

RESULTS: Extrapolating backwards in time, we found that differences in caudate and ventricular volumes between patients with HD and controls were evident 14 and 5 years, respectively, before motor onset $(P<.05)$. At onset, caudate volume was $2.58 \mathrm{~mL}$ smaller than that in controls $(P<.0001)$; ventricular volume was $9.27 \mathrm{~mL}$ larger $(P<.0001)$. HD caudate atrophy rates were linear, showed low variability between subjects, and were approximately 10 -fold higher than those in controls $(P<.001)$. $\mathrm{HD}$ ventricular enlargement rates were variable between subjects, were approximately 4-fold higher than those in controls at onset $(P<.001)$, and accelerated with disease duration $(P=.02)$.

CoNCLUSIONS: We provide evidence of acceleration of global atrophy in HD with disproportionate caudate involvement. Both caudate and global measures may be of use as early markers of HD pathology.

ABBREVIATIONS: $\mathrm{Cl}=$ confidence interval; Dur = disease duration (time since motor onset); $\mathrm{HD}=$ Huntington disease; ICC = intraclass correlation coefficient; $\mathrm{MBI}=$ mean brain intensity; $\mathrm{NHNN}=$ National Hospital for Neurology and Neurosurgery; NIHR = National Institute for Health Research; TIV = total intracranial volume; UCL = University College London; UHDRS = Unified Huntington's Disease Rating Scale

$\mathbf{H}^{\mathrm{D}}$ $\mathrm{D}$ is an autosomal dominantly inherited neurodegenerative disorder caused by a CAG repeat expansion in the gene encoding huntingtin. The disease is characterized by an insidious onset, followed by progressive cognitive decline,

Received October 6, 2009; accepted after revision November 27

From the Dementia Research Centre (N.Z.H., J.B., C.F., S.M.D.H., E.J.W., K.M., N.C.F.) and Department of Neurodegenerative Disease (N.Z.H., R.I.S., S.J.T.), UCL Institute of Neurology, University College London, United Kingdom; Medical Statistics Unit (C.F.), London School of Hygiene and Tropical Medicine, London, United Kingdom; Brain Repair Centre (R.A.B.), Department of Clinical Neurosciences, Addenbrooke's Hospital, Cambridge, United Kingdom; and Department of Clinical Neurology (N.C.F., S.J.T.), National Hospital for Neurology and Neurosurgery, London, United Kingdom.

This work was funded by the Cure Huntington's Disease Initiative Foundation (a not-forprofit research organization dedicated to HD). N.C.F. is funded by the Medical Research Council, United Kingdom. J.B. is funded by an Alzheimer's Research Trust United Kingdom research fellowship with the kind support of the Kirby Laing Foundation. This work was undertaken at University College London Hospital/UCL, which received a proportion of funding from the Department of Health's NIHR Biomedical Research Centres funding scheme. The Dementia Research Centre is an Alzheimer's Research Trust Coordinating Centre. This work was supported by an NIHR Biomedical Research Council award to Addenbrooke's Hospital.

N.C.F. and S.J.T. are equal senior authors.

Please address correspondence to N.Z. Hobbs, MEng, Dementia Research Centre, Box 16, National Hospital for Neurology and Neurosurgery, Queen Square, London WC1N 3BG, United Kingdom; e-mail: hobbs@drc.ion.ucl.ac.uk

Indicates open access to non-subscribers at www.ajnr.org

DOI 10.3174/ajnr.A2018 psychiatric disturbances, and motor impairment. Clinical (motor) onset is defined by the presence of unequivocal motor signs and usually occurs in mid-adulthood. Death typically occurs 15-20 years later.

There are currently no disease-modifying treatments that have been shown to slow the progression or delay the onset of HD in humans; however, several compounds have demonstrated promise in animal models of HD. ${ }^{1}$ Robust and sensitive markers of disease progression are required to assess their efficacy in humans. Current clinical measures are limited by floor and ceiling effects and lack sensitivity to change with time, especially in premanifest subjects who are likely to be the optimal candidates for therapeutic intervention.

Longitudinal MR imaging markers of progression have been used to assess treatment efficacy in neurodegenerative disorders such as Alzheimer disease ${ }^{2}$ and show promise for future clinical trials in $\mathrm{HD} .^{3,4}$ The caudate nucleus is of particular interest in HD because it is one of the earliest and most prominent sites of cell death. Caudate atrophy is evident some years before formal clinical diagnosis ${ }^{5,6}$ and increased rates of atrophy have been demonstrated in both premanifest and early HD relative to controls. ${ }^{7}$ However, extrastriatal degeneration is also evident early in the clinical course of $\mathrm{HD},{ }^{8-10}$ and a global marker of progression such as whole-brain atrophy ${ }^{3}$ or ventricular enlargement ${ }^{11}$ would have the advantage of en- 
compassing this diffuse atrophy in a single measure. However, global measures have not yet been shown to be sensitive markers of longitudinal change (when compared with controls) before clinical onset.

The aim of this study was to gain a better understanding of the structural changes occurring in the HD brain in relation to motor onset, both locally in the caudate and globally by using ventricular volumes. Specifically, we sought to determine the following: 1) the level of caudate atrophy and ventricular enlargement at recorded motor onset, 2) the rate and linearity of atrophy progression, and 3) an estimate of how many years before clinical onset volumes significantly deviate from those in controls. This information may facilitate future clinical trials in terms of subject selection and measurement of pathologic progression.

\section{Materials and Methods}

\section{Subjects}

Subjects with serial MR imaging were retrospectively selected from a larger study, ${ }^{12}$ provided they fulfilled any of the following criteria: 1 ) They had a diagnosis of early HD at the baseline assessment $(n=37)$, 2 ) they converted from premanifest HD to early HD during the study $(n=3)$, or 3$)$ they were healthy controls $(n=19)$. Early HD was defined as a diagnostic confidence score of 4 on the UHDRS, ${ }^{13}$ with a UHDRS total functional capacity score $\geq 7 .{ }^{14}$ Controls were partners or spouses of mutation carriers or individuals previously at risk for HD with a genetic test negative for the mutation.

Patients with HD had been recruited from the multidisciplinary HD clinic at the NHNN, London, and the Huntington's Disease Clinic at Addenbrooke's Hospital, Cambridge. All assessments were carried out at a single site (NHNN). No subjects were taking any medication known to influence brain volume, and subjects with concomitant neurologic illnesses were excluded.

\section{Ethics}

Subjects gave written informed consent in accordance with the Declaration of Helsinki, and the study had local research ethics committee and hospital trust approval.

\section{Image Acquisition and Processing}

Subjects were scanned at baseline, 12 months, and 27 months. Acquisition was consistent between subjects and time-points. Subjects underwent T1-weighted volumetric imaging on a 1.5T scanner using an inversion recovery prepared FAST spoiled gradient-recalled acquisition in steady-state sequence with a $24 \mathrm{~cm} \times 75 \%$ FOV and a $256 \times$ 256 matrix reconstructed to provide 124 contiguous $1.5-\mathrm{mm}$ thick coronal sections. In-plane pixel dimensions were $0.9375 \times 0.9375$ $\mathrm{mm}$. Acquisition parameters were as follows: $\mathrm{TR}=12 \mathrm{~ms}$, $\mathrm{TE}=5.2$ ms, flip angle $=13^{\circ}$, TI $=650 \mathrm{~ms}$, receiver bandwidth $=16 \mathrm{kHz}$, $\mathrm{NEX}=1$.

Image processing was performed by using Medical Image Display and Analysis Software. ${ }^{15}$ Brains were spatially normalized into Montreal Neurological Institute 305 atlas space ${ }^{16}$ with a 6- $d$ fregistration (3 translations, 3 rotations) to reduce the variability in landmark-derived arbitrary cutoffs used in the segmentation protocols. Repeat scans were coregistered to baseline by using an affine ( $12 d f$ ) ( 3 translations, 3 rotations, 3 scalings, and 3 shears) brain-brain registration. Scans for each subject were loaded on the monitor and segmented simultaneously by trained raters, with the rater blinded to time-point and diagnosis. Our segmentation protocols use semiautomated initialization steps based on consistent percentages of the MBI.

Caudate segmentation included the head and body of the caudate. $^{7}$ The rater manually positioned a "seed" (marker) inside the caudate, and intensity-threshold constraints at 69\% MBI and 110\% MBI formed an initial outline of the caudate. The rater then manually edited each section of the structure, which included over-riding the automated outline when necessary. To improve reproducibility, we manually removed the nucleus accumbens on the coronal view; inferiorally, segmentation stopped 1 section below the last section in which the ventricle was visible in the axial view. The slender caudate tail, a posterior extension of the caudate running parallel to the lateral ventricle, was excluded in the sagittal view while we ensured that the caudate remained continuous in the axial view. Caudate segmentations were carried out by 2 raters whose intra- and inter-rater reproducibility (ICCs) was $>0.99$ (mean absolute difference, $\sim 4 \%$ of the mean caudate volume).

Ventricular segmentation included the lateral ventricles and temporal horn of the lateral ventricles but not the third or fourth ventricles. ${ }^{17}$ A consistent threshold of $60 \%$ MBI provided an initial outline of the brain-CSF boundary of the ventricles. This was checked on every section and manually edited where necessary. Ventricular segmentation was carried out by 1 rater whose intrarater reproducibility (ICC) was $>0.99$ (mean absolute difference, $0.04 \%$ of mean ventricular volume).

TIV, an index of head size, was measured on each baseline scan by using a semiautomated protocol described by Whitwell et al. ${ }^{18} \mathrm{~A}$ lower threshold of $30 \% \mathrm{MBI}$ was used to outline the edge of the dura, which was manually edited when necessary. The inferior boundary was the lowest section in which cerebellar tissue was visible. Every tenth section was segmented, with the superior boundary being the highest section in which brain tissue was visible. A volume estimate was obtained by using linear interpolation. All TIV segmentations were carried out by 2 trained raters; intra- and inter-rater reproducibility (ICCs) was $>0.99$ (mean absolute difference, $<1 \%$ of mean TIV volume).

\section{Statistical Analysis}

Linear mixed models with random intercepts and fixed slopes were used to relate caudate and ventricular volumes in controls to age, adjusting for sex and TIV. Regression coefficients from these models were used to standardize caudate and ventricular volumes in patients with HD for sex and TIV. Illustrated results are those for a male subject with a TIV of $1500 \mathrm{~mL}$. Linear mixed models with random intercepts and fixed slopes were used to relate the age- and TIVadjusted volumes to time since motor onset (ie, disease duration). Further models of this type 1) included quadratic terms to investigate nonlinearity, 2) tested for heterogeneity in regression slopes, and 3) were adjusted for $C A G$ repeat length.

Regression coefficients from the normal aging models were also used to additionally standardize caudate volumes in patients with HD for normal aging. Linear mixed models with random intercepts and fixed slopes were used to relate these adjusted volumes to time since motor onset. From this model, the difference between mean volumes in patients with $\mathrm{HD}$ and controls at various disease durations (adjusting for age, sex, and TIV) was estimated. In testing for a reduction in volume relative to that in controls, 1 -sided $P$ values were appropriate. To give agreement with these 1 -sided $P$ values, we reported $90 \%$ CIs for differences. 


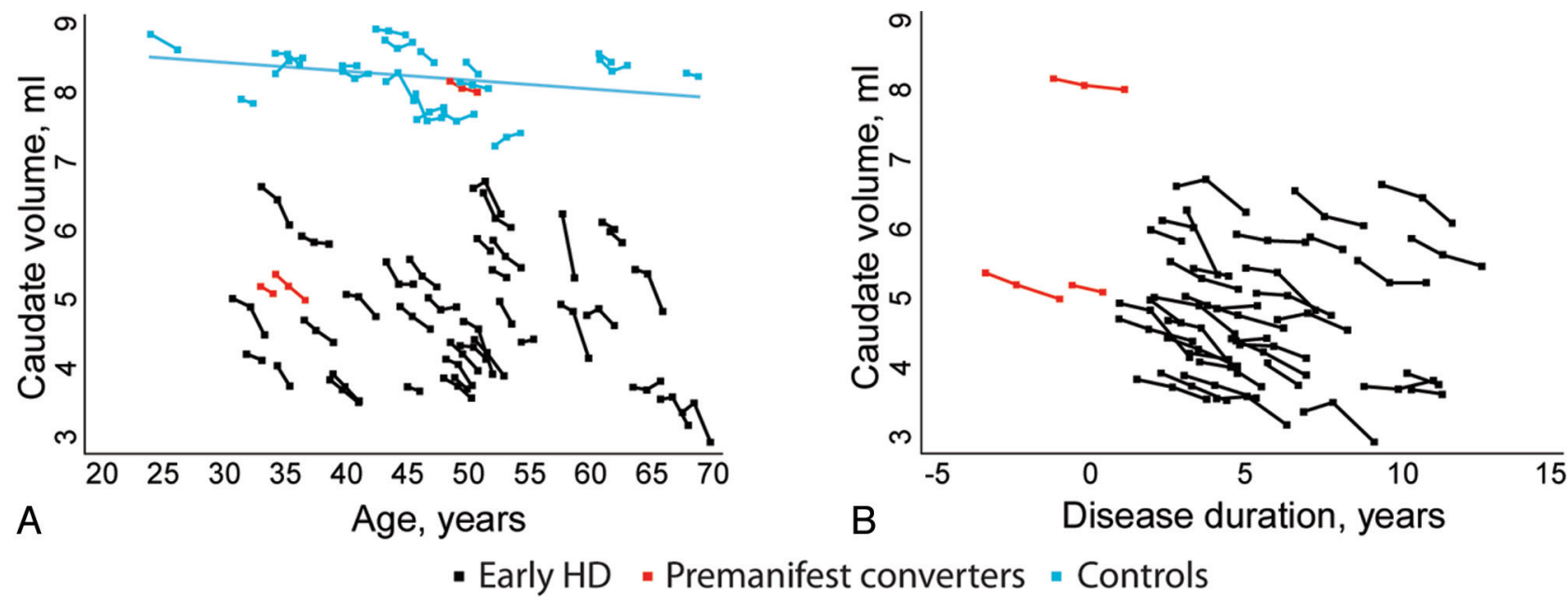

Fig 1. $A$, The relationship between caudate volume and age in controls and patients with HD standardized for sex (to a male) and head size (TIV $=1500 \mathrm{~mL}$ ). The blue line depicts the mean rate of normal aging. $B$, The relationship between caudate volume and disease duration (time since motor onset) standardized for sex (to a male) and head size (TIV $=1500 \mathrm{~mL}$ ). Standardization allows meaningful between-subject comparison and display.
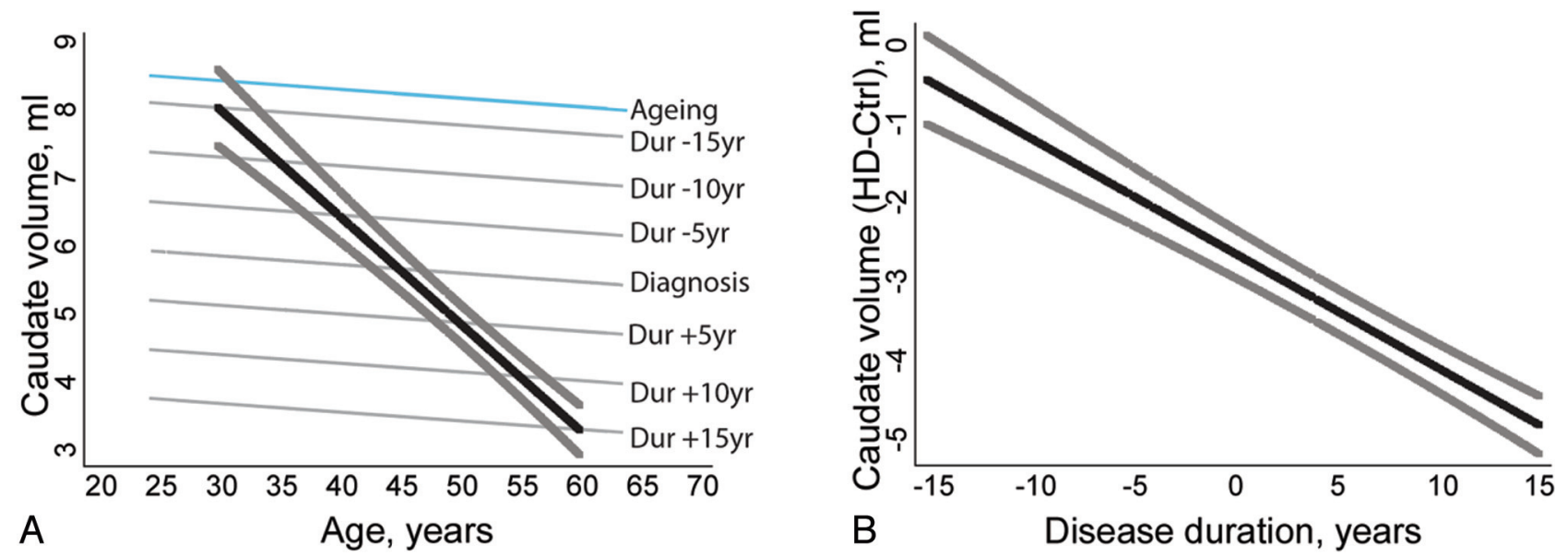

Fig 2. A, Illustration of the fitted relationship between caudate volume and age in normal aging (blue) and patients with HD according to disease duration (gray) for male subjects with a TIV of $1500 \mathrm{~mL}$. The black line shows the predicted trajectory (with $90 \% \mathrm{Cl}$ in thick gray) for a patient with $\mathrm{HD}$ with motor onset at 45 years of age. B, Fitted difference (with $90 \% \mathrm{CI}$ ) in caudate volume between patients with $\mathrm{HD}$ and controls according to disease duration. The difference is statistically significant $(P<.05,1$-sided) $\leq 14$ years before motor onset.

\section{Results}

Eighteen of the 19 control subjects who had baseline scans also were scanned at 12 months. The 1 control subject who missed the 12-month scanning returned at 27 months as did 12 others, giving a total of 50 scans. All 40 patients with HD had scans at baseline and 12 months, with 28 also being scanned at 27 months, a total of 108 scans. Three patients with HD were premanifest at baseline but experienced motor onset during $(n=2)$ or shortly after $(n=1)$ the study. The HD data ranged from 3.3 years before motor onset to 12.8 years post-motor onset. The mean age at baseline in controls was $45.6 \pm 10.7$ years; range, $24.4-68.3$ years; and in $\mathrm{HD}$, the mean was $48.6 \pm$ 10.0 years; range, $31.2-68.0$ years. There was no significant difference in age between groups $(P=.3)$. Mean $C A G$ repeat length in the HD group was $43.5 \pm 2.3$; range, $40-50$.

\section{Caudate Atrophy}

In controls, there was a small and nonsignificant effect of age on caudate volume, with caudate volumes decreasing, on average, by $0.013 \mathrm{~mL}$ (95\% CI, -0.006 to $0.032 \mathrm{~mL}$ ) per year of normal aging after adjusting for sex and TIV (Fig 1A).
In the HD group, there was a strong linear effect of disease duration (which here includes the effect of normal aging) on caudate volume, with volumes decreasing by $0.158 \mathrm{~mL}$ per year (95\% CI, $0.129-0.187 \mathrm{~mL}$ per year; $P<.0001$ ) after adjusting for age and TIV (Fig $1 B$ ). Using a model adjusted for normal aging, we estimated HD caudate volumes to decrease, on average by $0.146 \mathrm{~mL}$ per year $(95 \% \mathrm{CI}, 0.117-0.175 \mathrm{~mL}$ per year, $P<.0001)$ more than those of controls of the same age, sex, and TIV. There was no evidence that longitudinal caudate volume change was nonlinear, nor was there evidence of variability in atrophy rates among patients with HD.

At motor onset, mean total caudate volume in patients with HD was, on average, $2.58 \mathrm{~mL}$ (95\% CI, 2.21-2.95 mL; $P<$ $.0001)$ lower than that in controls of the same age and sex with the same TIV (Figs $1 B$ and $2 B$ ). Between-subject variability in cross-sectional caudate volumes was higher in patients with $\mathrm{HD}(\mathrm{SD}=1.05 \mathrm{~mL})$ than in controls $(\mathrm{SD}=0.45 \mathrm{~mL})$, even after accounting for disease duration, TIV, sex, and age. The addition of $C A G$ to the model only had a small effect on between-subject variability, reducing the SD from 1.05 to 0.99 $\mathrm{mL}$. 


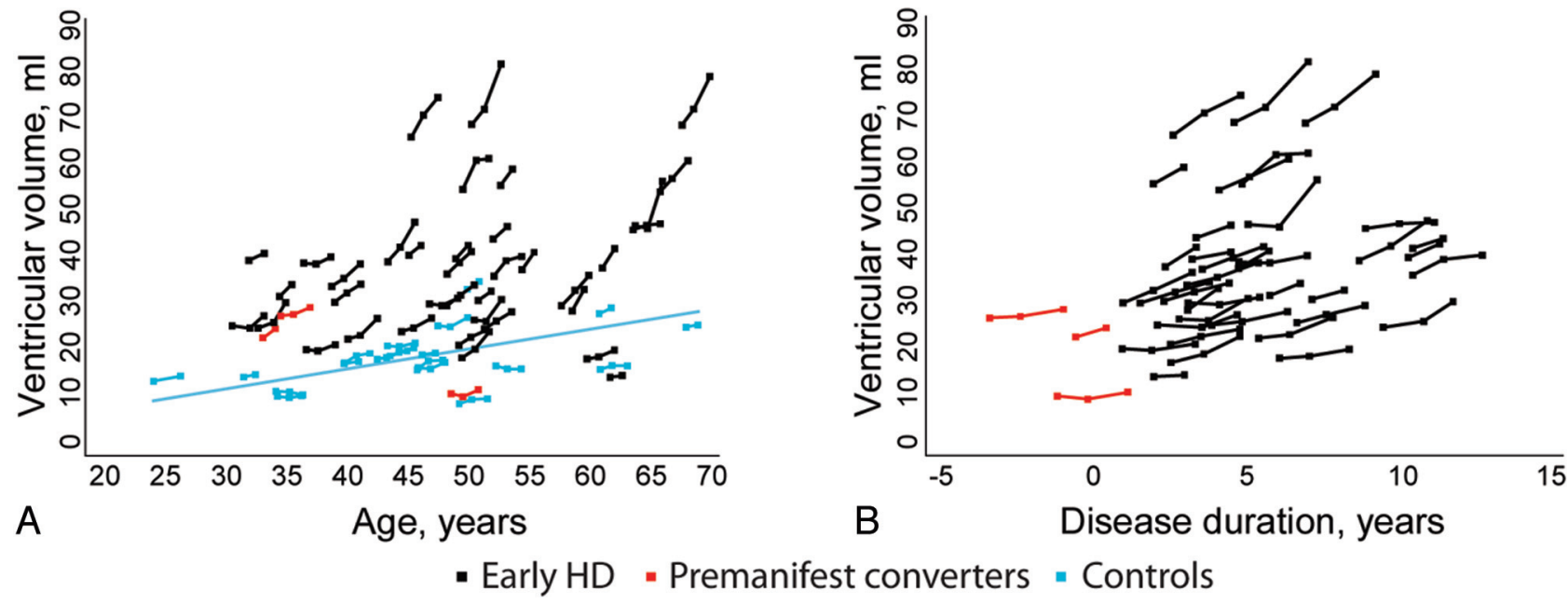

Fig 3. $A$, The relationship between ventricular volume and age in controls and patients with HD standardized for sex (to a male) and head size (TIV $=1500 \mathrm{~mL}$ ). The blue line depicts the mean rate of normal aging. $B$, The relationship between ventricular volume and HD disease duration (time since motor onset) standardized for sex (to a male) and head size (TIV $=$ $1500 \mathrm{~mL})$.
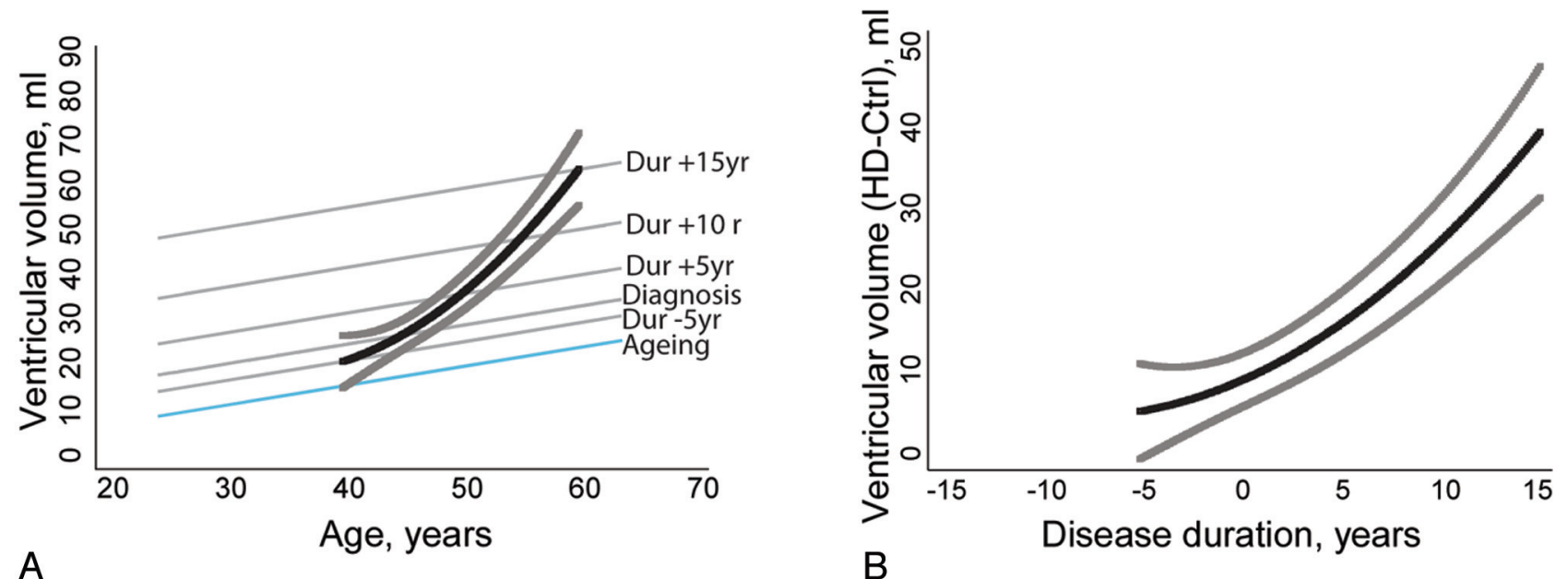

Fig 4. A, Illustration of the fitted relationship between ventricular volume and age in normal aging (blue) and patients with HD according to disease duration (gray) for male subjects with a TIV of $1500 \mathrm{~mL}$. The black line shows the predicted trajectory (with $90 \% \mathrm{Cl}$ in thick gray) for a patient with $\mathrm{HD}$ with motor onset at 45 years of age. B, Fitted difference (with $90 \% \mathrm{CI}$ ) in ventricular volume between patients with $\mathrm{HD}$ and controls according to disease duration. The difference is statistically significant $(P<.05,1$-sided $) \leq 5$ years before motor onset.

By linearly extrapolating from the model, adjusting the reductions in caudate volume for normal aging, we estimated caudate volume to deviate significantly from normal $\leq 14$ years before motor onset $(P<.05$, 1-tailed; Fig 2$)$.

\section{Ventricular Enlargement}

In controls, there was a significant linear effect of age on ventricular volume, with volumes increasing by $0.43 \mathrm{~mL}$ per year (95\% CI, $0.27-0.60 \mathrm{~mL}$ per year; $P<.0001$ ) after adjusting for sex and TIV (Fig $3 A$ ).

In the HD group, there was evidence of heterogeneity in ventricular enlargement rates $(P<.0001)$ and of a year-onyear acceleration in ventricular enlargement $(P=.02)$, after adjusting for sex and TIV. These features were accommodated by including random slopes and (fixed effect) quadratic terms in the linear mixed models. Ventricular volume was estimated to increase by $1.44 \mathrm{~mL}$ ( $95 \% \mathrm{CI}, 0.71-2.16 \mathrm{~mL}$ ) in the year immediately before disease onset and by $1.57 \mathrm{~mL}$ (95\% CI, 0.93-2.20 $\mathrm{mL}$ ) in the year immediately after onset; the differ- ence was explained by the acceleration of $0.13 \mathrm{~mL}$ per year ${ }^{2}$ (95\% CI, 0.024-0.24 $\mathrm{mL}$ per year ${ }^{2} ; P=.02$ ).

Using a model adjusted for normal aging, sex, and TIV, we estimated $\mathrm{HD}$ ventricular volume to increase by $1.01 \mathrm{~mL}(95 \%$ $\mathrm{CI}, 0.23-1.79 \mathrm{~mL}$ ) more than that in controls in the year before motor onset and by $1.14 \mathrm{~mL}$ (95\% CI, 0.46-1.82 mL) more than that in controls in the year following onset, an acceleration of $0.13 \mathrm{~mL}$ per year ${ }^{2}$ (95\% CI, $0.013-0.24 \mathrm{~mL}$ per year ${ }^{2}$; $P=.03)$.

Mean ventricular volume at motor onset was estimated to be, on average, $9.27 \mathrm{~mL}$ (95\% CI, 5.29-13.26 mL, $P<.0001$ ) larger in patients with $\mathrm{HD}$ than controls of the same age and sex with the same TIV (Fig 4A,-B). Predicted between-subject variability in ventricular volume was $10.9 \mathrm{~mL}$ at onset and increased with disease duration to a value of $18.7 \mathrm{~mL}$ at a duration of 10 years. Between-subject variability was $6.00 \mathrm{~mL}$ in controls.

Figure $4 B$ displays the estimated difference in mean ventricular volume between patients with HD and controls of the 
same age, sex, and TIV, according to time from motor onset. If one extrapolates backwards in time, the difference is statistically significant $\leq 4.7$ years before motor onset $(P<.05$, 1-tailed).

\section{Discussion}

In this serial MR imaging study, we used reliable volumetric measurements and actual (rather than predicted) dates of motor onset to assess how caudate and global volumes change as HD progresses from premanifest to early clinical disease. $\mathrm{Pa}-$ tients with HD of the same disease duration showed heterogeneity in cross-sectional caudate and ventricular volumes despite adjustment for confounding factors. HD caudate atrophy rates were linear, showed low variability among subjects, and were approximately 10-fold higher than those in controls. HD ventricular enlargement rates were variable among subjects, were approximately 4-fold higher than those in controls at motor onset, and accelerated with disease duration. We estimated HD caudate and ventricular volumes to deviate from normal approximately 14 and 5 years, respectively, before actual motor onset. Using data from premanifest subjects who underwent motor onset during our study, combined with data from early clinical subjects who had dates of onset recorded, has allowed us to predict with greater accuracy when structural brain changes occur in the preclinical phase of HD.

A notable finding from this study was the high variability in cross-sectional caudate and ventricular volume among patients with $\mathrm{HD}$ of the same disease duration despite adjustment for age, sex, TIV, and CAG repeat length. This suggests that the current way of defining clinical onset, according to the presence of unequivocal motor signs, may not have a close temporal relationship with atrophy or that there are other unknown determinants of volume and/or atrophy such as genetic and environmental factors that play an important role. Heterogeneity in cross-sectional volumes highlights the importance of longitudinal studies in which subjects act as their own controls, thus reducing the problems of between-subject variability.

By combining our cross-sectional and longitudinal data, we estimated caudate volumes to deviate from normal approximately 14 years before motor onset, with volumes reduced by the order of $30 \%$ at onset. Caudate atrophy rates were linear, with no evidence of acceleration, and were approximately 10-fold higher in patients than in controls. Importantly, there was low variability in caudate atrophy rates among patients regardless of their disease duration. These findings suggest that longitudinal change in caudate volume would be an appropriate marker of HD pathology several years before onset. Our findings confirm previous work by Aylward et $\mathrm{al},{ }^{6}$ which demonstrated a linear decline in caudate volume with disease progression and suggested that caudate volume may deviate from normal between 9 and 20 years from predicted motor onset. A cross-sectional study of premanifest subjects only (no controls) estimated striatal atrophy to begin approximately 15 years before estimated motor onset, with a short nonlinear transition period followed by a fairly linear decline in volume. ${ }^{5}$ Although both of these studies included predicted dates of motor onset, an imperfect proxy for actual dates, our overall findings are consistent. There is a consensus that caudate losses are evident approximately 15 years before motor onset of HD and that caudate atrophy progresses in a linear and relatively consistent manner between patients.

We estimated ventricular volume to deviate from normal approximately 5 years before motor onset, approximately a decade later than that in the caudate. This reduced sensitivity is most likely caused by the high natural variability in the ventricles masking the earliest volumetric losses, which are subtle and mainly confined to the striatum. At motor onset, ventricular enlargement rates were approximately 4-fold higher in patients with HD than in controls, which is consistent with the findings of Squitieri et al. ${ }^{11}$ Additionally we found evidence of a significant year-on-year acceleration in ventricular enlargement. This acceleration most likely reflects the known extension of pathology to extrastriatal gray matter and white matter regions as the disease progresses. ${ }^{8,10,19}$ Nonlinearity of change and insensitivity to early pathology are not favorable characteristics for a biomarker. However, global markers of loss will better capture the totality of neurodegeneration and, as a marker of therapeutic efficacy, will allow assessment of disease-slowing effects outside the caudate. This may be valuable given the difficulty of predicting the location of therapeutic effect.

Significantly increased global atrophy rates have not been demonstrated before the clinical (motor) onset of HD in patients with HD compared with healthy controls. ${ }^{3,11}$ However, our results suggest that with careful selection of subjects relatively close to motor onset on the basis of their CAG repeat size and age, ${ }^{20}$ it should be possible to measure longitudinal changes in global pathology within 5 years of motor onset. Furthermore, although the ventricles appear to be a less sensitive measure than the caudate, they have the benefit of well-defined anatomic boundaries, which make volumetric measurement quicker and more reliable. Importantly, ventricular enlargement may not be a perfect indicator of global atrophy in HD because caudate atrophy will have a disproportionate effect on ventricular enlargement due to their shared boundaries. Therefore, using ventricular volume as a global measure may result in slightly more pronounced control-HD differences than would be apparent with a measure such as whole-brain volume.

This study is strengthened by the use of actual rather than predicted dates of motor onset. The use of predicted dates can add considerable error to this type of analysis, and this is highlighted by our 3 premanifest converters who underwent onset when they were approximately 10, 11, and 17 years from a predicted $60 \%$ probability of onset based on the survival model of Langbehn et al. ${ }^{20}$ In fact, in our larger study, we followed 21 premanifest subjects; however, for this analysis, we chose to exclude subjects who had not undergone motor onset because we thought that the errors associated with predicting time to onset outweighed the advantages of having more data on premanifest atrophy. However, we acknowledge that by doing this, we have limited our analysis to small numbers and a relatively short time range preonset. Making predictions concerning differences in caudate volumes between patients with HD and controls many years before onset requires us to make assumptions concerning the linearity of atrophy rates before our follow-up time. Therefore, our observation that the difference in caudate volumes was statistically 
significant $\leq 14$ years before motor onset should be interpreted with some caution. Nonetheless, the linearity of the volume changes during the follow-up period can give us some confidence in this prediction.

A strength of this study is the level of care taken with the volumetric measurements. By segmenting scans for each subject side by side, blinded to time-point, we ensured that segmentations were anatomically consistent with respect to cutoffs. This resulted in precise volume measurements at all 3 time-points, enabling us to reliably assess and model volume change in a relatively small cohort. Our retrospective study design also reduced the effects of "segmentor drift," an increase in intrarater variation with time, which can confound longitudinal volumetric measurements.

\section{Conclusions}

In summary, this study provides evidence of acceleration of global atrophy in HD with disproportionate caudate involvement. Longitudinal change in caudate volume was linear and consistent among patients with HD and, hence, could provide a sensitive and robust marker of pathology from many years before onset. In contrast, our global marker (ventricular volume change) showed heterogeneity in rates of change and was relatively insensitive to early volumetric losses. This measure may prove useful as a marker of pathology in subjects relatively close to motor onset.

\section{Acknowledgments}

We thank the patients and controls who took part in this study.

\section{References}

1. Handley OJ, Naji JJ, Dunnett SB, et al. Pharmaceutical, cellular and genetic therapies for Huntington's disease. Clin Sci (Lond) 2006;110:73-88

2. Fox NC, Black RS, Gilman S, et al. Effects of Abeta immunization (AN1792) on MRI measures of cerebral volume in Alzheimer disease. Neurol 2005;64:1563-72

3. Henley SM, Wild EJ, Hobbs NZ, et al. Whole-brain atrophy as a measure of progression in premanifest and early Huntington's disease. Mov Disord 2009;2:932-36

4. Aylward EH. Change in MRI striatal volumes as a biomarker in preclinical Huntington's disease. Brain Res Bull 2007;72:152-58

5. Paulsen JS, Hayden M, Stout JC, et al. Preparing for preventive clinical trials: the Predict-HD study. Arch Neurol 2006;63:883-90

6. Aylward EH, Sparks BF, Field KM, et al. Onset and rate of striatal atrophy in preclinical Huntington disease. Neurol 2004;63:66-72

7. Hobbs NZ, Henley SM, Wild EJ, et al. Automated quantification of caudate atrophy by local registration of serial MRI: evaluation and application in Huntington's disease. Neuroimage 2009;47:1659-65

8. Rosas HD, Salat DH, Lee SY, et al. Cerebral cortex and the clinical expression of Huntington's disease: complexity and heterogeneity. Brain 2008; 131:1057-68

9. Ruocco HH, Lopes-Cendes I, Li LM, et al. Striatal and extrastriatal atrophy in Huntington's disease and its relationship with length of the CAG repeat. Braz J Med Biol Res 2006;39:1129-36

10. Ciarmiello A, Cannella M, Lastoria S, et al. Brain white-matter volume loss and glucose hypometabolism precede the clinical symptoms of Huntington's disease. J Nucl Med 2006;47:215-22

11. Squitieri F, Cannella M, Simonelli M, et al. Distinct brain volume changes correlating with clinical stage, disease progression rate, mutation size, and age at onset prediction as early biomarkers of brain atrophy in Huntington's disease. CNS Neurosci Ther 2009;15:1-11

12. Henley SM, Wild EJ, Hobbs NZ, et al. Defective emotion recognition in early HD is neuropsychologically and anatomically generic. Neuropsychologia 2008; 46:2152-60. Epub 2008 Mar 6

13. Unified Huntington's Disease Rating Scale: reliability and consistencyHuntington Study Group. Mov Disord 1996;11:136-42

14. Shoulson I, Fahn S. Huntington disease: clinical care and evaluation. Neurology 1979;29:1-3

15. Freeborough PA, Fox NC, Kitney RI. Interactive algorithms for the segmentation and quantitation of 3-D MRI brain scans. Comput Methods Programs Biomed 1997;53:15-25

16. Mazziotta JC, Toga AW, Evans A, et al. A probabilistic atlas of the human brain: theory and rationale for its development-The International Consortium for Brain Mapping (ICBM). Neuroimage 1995;2:89-101

17. Scahill RI, Frost C, Jenkins R, et al. A longitudinal study of brain volume changes in normal aging using serial registered magnetic resonance imaging. Arch Neurol 2003;60:989-94

18. Whitwell JL, Crum WR, Watt HC, et al. Normalization of cerebral volumes by use of intracranial volume: implications for longitudinal quantitative MR imaging. AJNR Am J Neuroradiol 2001;22:1483-89

19. Fennema-Notestine C, Archibald SL, Jacobson MW, et al. In vivo evidence of cerebellar atrophy and cerebral white matter loss in Huntington disease. $\mathrm{Neu}$ rology 2004;63:989-95

20. Langbehn DR, Brinkman RR, Falush D, et al. A new model for prediction of the age of onset and penetrance for Huntington's disease based on CAG length. Clin Genet 2004;65:267-77 